\title{
Article \\ Fractional Integral of a Confluent Hypergeometric Function Applied to Defining a New Class of Analytic Functions
}

\author{
Alina Alb Lupaş *, + (i) and Georgia Irina Oros ${ }^{+}($i) \\ Department of Mathematics and Computer Science, University of Oradea, 1 Universitatii Street, \\ 410087 Oradea, Romania; georgia_oros_ro@yahoo.co.uk \\ * Correspondence: alblupas@gmail.com or dalb@uoradea.ro \\ + These authors contributed equally to this work.
}

check for updates

Citation: Alb Lupaş, A.; Oros, G.I. Fractional Integral of a Confluent Hypergeometric Function Applied to Defining a New Class of Analytic Functions. Symmetry 2022, 14, 427. https://doi.org/10.3390/ sym 14020427

Academic Editor: Stanisława Kanas

Received: 4 February 2022

Accepted: 19 February 2022

Published: 21 February 2022

Publisher's Note: MDPI stays neutral with regard to jurisdictional claims in published maps and institutional affiliations.

Copyright: () 2022 by the authors. Licensee MDPI, Basel, Switzerland. This article is an open access article distributed under the terms and conditions of the Creative Commons Attribution (CC BY) license (https:// creativecommons.org/licenses/by/ $4.0 /)$.

\begin{abstract}
The study on fractional integrals of confluent hypergeometric functions provides interesting subordination and superordination results and inspired the idea of using this operator to introduce a new class of analytic functions. Given the class of functions $\mathcal{A}_{n}=\{f \in \mathcal{H}(U): f(z)=z+$ $\left.a_{n+1} z^{n+1}+\ldots, z \in U\right\}$ written simply $\mathcal{A}$ when $n=1$, the newly introduced class involves functions $f \in \mathcal{A}$ considered in the study due to their special properties. The aim of this paper is to present the outcomes of the study performed on the new class, which include a coefficient inequality, a distortion theorem and extreme points of the class. The starlikeness and convexity properties of this class are also discussed, and partial sums of functions from the class are evaluated in order to obtain class boundary properties.
\end{abstract}

Keywords: analytic function; coefficient inequality; partial sum; starlike function; convex function; fractional integral; confluent hypergeometric function

\section{Introduction}

The study presented in this paper is done in the general, well-known space on Geometric Function Theory. The main notions and notations are recalled. The unit disc of the complex plane is denoted by $U=\{z \in \mathbb{C}:|z|<1\}$. $\mathcal{H}(U)$ refers to the class of analytic functions in the unit disc, and one of its remarkable subclasses is denoted by $\mathcal{A}_{n}=\left\{f \in \mathcal{H}(U), f(z)=z+a_{n+1} z^{n+1}+\ldots, z \in U\right\}, a \in \mathbb{C}$ and $n \in \mathbb{N}$, with $\mathcal{A}_{1}$ written simply $\mathcal{A}$.

The special class of starlike functions of order $\alpha$ is defined as

$$
\mathcal{S}^{*}(\alpha)=\left\{f \in \mathcal{A}: \operatorname{Re} \frac{z f^{\prime}(z)}{f(z)}>\alpha, 0<\alpha<1\right\},
$$

and the class of convex functions of order $\alpha$ is defined as

$$
\mathcal{K}(\alpha)=\left\{f \in \mathcal{A}: \operatorname{Re}\left(\frac{z f^{\prime \prime}(z)}{f^{\prime}(z)}+1\right)>\alpha, 0<\alpha<1\right\} .
$$

For $n$ as a positive integer and $a$ as a complex number,

$$
\mathcal{H}[a, n]=\left\{f \in \mathcal{H}(U): f(z)=a+a_{n} z^{n}+z_{n+1} z^{n+1}+\ldots, z \in U\right\},
$$

with $\mathcal{H}_{0}=\mathcal{H}[0,1]$.

Operators have been used from the beginning of the study of complex-valued functions because they make it possible to elegantly obtain new and remarkable outcomes and to give easier proofs for known results. Recently, many new operators [1,2] were defined with the help of the fractional integral of order $\lambda$ previously used by Owa and Srivastava $[3,4]$. 
Definition $1([3,4])$. The fractional integral of order $\lambda(\lambda>0)$ is defined for a function $f$ by

$$
D_{z}^{-\lambda} f(z)=\frac{1}{\Gamma(\lambda)} \int_{0}^{z} \frac{f(t)}{(z-t)^{1-\lambda}} d t
$$

where $f$ is an analytic function in a simply-connected region of the z-plane containing the origin, and the multiplicity of $(z-t)^{\lambda-1}$ is removed by requiring $\log (z-t)$ to be real, when $(z-t)>0$.

Inspired by earlier work done by applying this function on different hypergeometric functions seen in papers, such as $[5,6]$, a new operator was defined combining confluent hypergeometric function with the remarkable fractional integral in [7]. The new operator was introduced with inspiration from the numerous studies conducted on confluent hypergeometric functions from different perspectives, such as using it together with other remarkable functions (for example in $[8,9]$ ) or studying its univalence properties as seen in $[10,11]$.

The definition of the confluent (Kummer) hypergeometric function was found, for example, in [12].

Definition 2 ([12] (p. 5)). Let a and $c$ be complex numbers with $c \neq 0,-1,-2, \ldots$ and consider

$$
\phi(a, c ; z)={ }_{1} F_{1}(a, c ; z)=1+\frac{a}{c} \frac{z}{1 !}+\frac{a(a+1)}{c(c+1)} \frac{z^{2}}{2 !}+\ldots, z \in U .
$$

This function is called a confluent (Kummer) hypergeometric function, is analytic in $\mathbb{C}$ and satisfies Kummer's differential equation

$$
z w^{\prime \prime}(z)+(c-z) w^{\prime}(z)-a w(z)=0 .
$$

Considering

$$
(d)_{k}=\frac{\Gamma(d+k)}{\Gamma(d)}=d(d+1)(d+2) \ldots(d+k-1) \text { and }(d)_{0}=1,
$$

the confluent (Kummer) hypergeometric function has the following form

$$
\phi(a, c ; z)=\sum_{k=0}^{\infty} \frac{(a)_{k}}{(c)_{k}} \frac{z^{k}}{k !}=\frac{\Gamma(c)}{\Gamma(a)} \sum_{k=0}^{\infty} \frac{\Gamma(a+k)}{\Gamma(c+k)} \frac{z^{k}}{k !} .
$$

The definition of the operator used for obtaining the original results presented in this paper, defined in [7] as previously mentioned, is shown in the next definition.

Definition 3 ([7]). Let $a$ and $c$ be complex numbers with $c \neq 0,-1,-2, \ldots$ and $\lambda>0$. We define the fractional integral of the confluent hypergeometric function

$$
\begin{gathered}
D_{z}^{-\lambda} \phi(a, c ; z)=\frac{1}{\Gamma(\lambda)} \int_{0}^{z} \frac{\phi(a, c ; t)}{(z-t)^{1-\lambda}} d t \\
=\frac{1}{\Gamma(\lambda)} \frac{\Gamma(c)}{\Gamma(a)} \sum_{k=0}^{\infty} \frac{\Gamma(a+k)}{\Gamma(c+k) \Gamma(k+1)} \int_{0}^{z} \frac{t^{k}}{(z-t)^{1-\lambda}} d t .
\end{gathered}
$$

The fractional integral of confluent hypergeometric function can be written

$$
D_{z}^{-\lambda} \phi(a, c ; z)=\frac{\Gamma(c)}{\Gamma(a)} \sum_{k=0}^{\infty} \frac{\Gamma(a+k)}{\Gamma(c+k) \Gamma(\lambda+k+1)} z^{k+\lambda},
$$

after a simple calculation. Evidently, $D_{z}^{-\lambda} \phi(a, c ; z) \in \mathcal{H}[0, \lambda]$. 
We next introduce a new operator defined by convolution of the operator seen in Definition 3 and a function $f \in \mathcal{A}$.

Definition 4. Denote by $D_{z}^{-\lambda} \Phi(a, c)$ the operator given by the convolution of the operator $D_{z}^{-\lambda} \phi(a, c ; z)$ given by (4) and the analytic function $f \in \mathcal{A}, D_{z}^{-\lambda} \Phi(a, c): \mathcal{A} \rightarrow \mathcal{A}$,

$$
D_{z}^{-\lambda} \Phi(a, c)(z)=D_{z}^{-\lambda} \phi(a, c ; z) * f(z)
$$

Remark 1. For $f \in \mathcal{A}, f(z)=z+\sum_{k=2}^{\infty} a_{k} z^{k}$, we find

$$
D_{z}^{-\lambda} \Phi(a, c)(z)=z+\frac{\Gamma(c)}{\Gamma(a)} \sum_{k=2}^{\infty} \frac{\Gamma(a+k)}{\Gamma(c+k) \Gamma(\lambda+k+1)} a_{k+\lambda} z^{k+\lambda}
$$

Inspired by the paper published by A.R. Juma and H. Ziraz [13], the new class of analytic functions is defined as:

Definition 5. Denote by $\mathcal{D} \oplus(d, \alpha, \beta, a, c, \lambda)$ the class consisting of functions $f \in \mathcal{A}$ that satisfy the inequality:

$$
\left|\frac{1}{d}\left(\frac{z\left(D_{z}^{-\lambda} \Phi(a, c)(z)\right)^{\prime}+\alpha z^{2}\left(D_{z}^{-\lambda} \Phi(a, c)(z)\right)^{\prime \prime}}{(1-\alpha) D_{z}^{-\lambda} \Phi(a, c)(z)+\alpha z\left(D_{z}^{-\lambda} \Phi(a, c)(z)\right)^{\prime}}-1\right)\right|<\beta,
$$

where $z \in U, d \in \mathbb{C}-\{0\}, 0<\beta \leq 1,0 \leq \alpha \leq 1, a, c \in \mathbb{C}, c \neq 0,-1,-2, \ldots, \lambda>0$.

For the study done on this class, we also considered recently published results, such as the geometric properties proved by the authors related to classes of $p$-valent functions [14], $q$-starlike functions involving Janowski functions [15] or harmonic $p$-valent functions based on hypergeometric functions [16].

The first original results related to the class $\mathcal{D} \oplus(d, \alpha, \beta, a, c, \lambda)$ are presented in the next section regarding conditions that are necessary and sufficient such that function $f \in \mathcal{A}$ belongs to the class. Furthermore, coefficient-related studies establish certain relevant inequalities in Section 2, and distortion properties for functions from class $\mathcal{D} \oplus(d, \alpha, \beta, a, c, \lambda)$ and for their derivatives are presented in Section 3. Partial sums of functions from class $\mathcal{D} \oplus(d, \alpha, \beta, a, c, \lambda)$ are considered in Section 4, and the extreme points are found for the class. In Section 5, certain inclusion properties are proven for class $\mathcal{D} \oplus(d, \alpha, \beta, a, c, \lambda)$, and finally, aspects regarding the starlikeness and convexity properties of the class are discussed in Section 6 of the paper.

\section{Study Regarding Function Class Coefficients}

Conditions that must be complied by functions in order to belong to class $\mathcal{D} \oplus(d, \alpha$, $\beta, a, c, \lambda)$ are first established.

Theorem 1. A certain function $f \in \mathcal{A}$ is part of class $\mathcal{D} \oplus(d, \alpha, \beta, a, c, \lambda)$ if and only if the next inequality holds:

$$
\sum_{k=2}^{\infty} \frac{\Gamma(a+k)}{\Gamma(c+k) \Gamma(\lambda+k+1)}(k+\lambda-1+\beta|d|)[1+\alpha(k+\lambda-1)] a_{k+\lambda} \leq \beta|d| \frac{\Gamma(a)}{\Gamma(c)},
$$

where $z \in U, d \in \mathbb{C}-\{0\}, 0<\beta \leq 1,0 \leq \alpha \leq 1, a, c \in \mathbb{C}, c \neq 0,-1,-2, \ldots, \lambda>0$.

Proof. Suppose that function $f \in \mathcal{A}$ and that inequality (6) holds. Then, we obtain

$$
\left|\frac{z\left(D_{z}^{-\lambda} \Phi(a, c)(z)\right)^{\prime}+\alpha z^{2}\left(D_{z}^{-\lambda} \Phi(a, c)(z)\right)^{\prime \prime}}{(1-\alpha) D_{z}^{-\lambda} \Phi(a, c)(z)+\alpha z\left(D_{z}^{-\lambda} \Phi(a, c)(z)\right)^{\prime}}-1\right|=
$$




$$
\begin{aligned}
& \left|\frac{z+\frac{\Gamma(c)}{\Gamma(a)} \sum_{k=2}^{\infty} \frac{\Gamma(a+k)}{\Gamma(c+k) \Gamma(\lambda+k+1)}(k+\lambda) a_{k+\lambda} z^{k+\lambda}+\alpha \frac{\Gamma(c)}{\Gamma(a)} \sum_{k=2}^{\infty} \frac{\Gamma(a+k)}{\Gamma(c+k) \Gamma(\lambda+k+1)}(k+\lambda)(k+\lambda-1) a_{k+\lambda} z^{k+\lambda}}{(1-\alpha) z+(1-\alpha) \frac{\Gamma(c)}{\Gamma(a)} \sum_{k=2}^{\infty} \frac{\Gamma(a+k)}{\Gamma(c+k) \Gamma(\lambda+k+1)} a_{k+\lambda} z^{k+\lambda}+\alpha z+\alpha \frac{\Gamma(c)}{\Gamma(a)} \sum_{k=2}^{\infty} \frac{\Gamma(a+k)}{\Gamma(c+k) \Gamma(\lambda+k+1)}(k+\lambda) a_{k+\lambda} z^{k+\lambda}}-1\right|= \\
& \left|\frac{\sum_{k=2}^{\infty} \frac{\Gamma(a+k)}{\Gamma(c+k) \Gamma(\lambda+k+1)}(k+\lambda-1)[1+\alpha(k+\lambda-1)] a_{k+\lambda} z^{k+\lambda}}{\frac{\Gamma(a)}{\Gamma(c)} z+\sum_{k=2}^{\infty} \frac{\Gamma(a+k)}{\Gamma(c+k) \Gamma(\lambda+k+1)}[1+\alpha(k+\lambda-1)] a_{k+\lambda} z^{k+\lambda}}\right| \\
& \leq \frac{\sum_{k=2}^{\infty} \frac{\Gamma(a+k)}{\Gamma(c+k) \Gamma(\lambda+k+1)}(k+\lambda-1)[1+\alpha(k+\lambda-1)] a_{k+\lambda}|z|^{k+\lambda-1}}{\frac{\Gamma(a)}{\Gamma(c)}-\sum_{k=2}^{\infty} \frac{\Gamma(a+k)}{\Gamma(c+k) \Gamma(\lambda+k+1)}[1+\alpha(k+\lambda-1)] a_{k+\lambda}|z|^{k+\lambda-1}}<\beta|d| .
\end{aligned}
$$

Considering for $z$ values on the real axis and putting $z \rightarrow 1^{-}$, we have

$$
\sum_{k=2}^{\infty} \frac{\Gamma(a+k)}{\Gamma(c+k) \Gamma(\lambda+k+1)}(k+\lambda-1+\beta|d|)[1+\alpha(k+\lambda-1)] a_{k+\lambda} \leq \beta|d| \frac{\Gamma(a)}{\Gamma(c)} .
$$

Conversely, supposing $f(z) \in \mathcal{D} \oplus(d, \alpha, \beta, a, c, \lambda)$, we obtain the following inequality

$$
\begin{gathered}
\operatorname{Re}\left\{\left|\frac{z\left(D_{z}^{-\lambda} \Phi(a, c)(z)\right)^{\prime}+\alpha z^{2}\left(D_{z}^{-\lambda} \Phi(a, c)(z)\right)^{\prime \prime}}{(1-\alpha) D_{z}^{-\lambda} \Phi(a, c)(z)+\alpha z\left(D_{z}^{-\lambda} \Phi(a, c)(z)\right)^{\prime}}-1\right|\right\}-\beta|d| \\
\operatorname{Re}\left\{\frac{z+\frac{\Gamma(c)}{\Gamma(a)} \sum_{k=2}^{\infty} \frac{\Gamma(a+k)}{\Gamma(c+k) \Gamma(\lambda+k+1)}(k+\lambda)[1+\alpha(k+\lambda-1)] a_{k+\lambda} z^{k+\lambda}}{z+\frac{\Gamma(c)}{\Gamma(a)} \sum_{k=2}^{\infty} \frac{\Gamma(a+k)}{\Gamma(c+k) \Gamma(\lambda+k+1)}[1+\alpha(k+\lambda-1)] a_{k+\lambda} z^{k+\lambda}}-1+\beta|d|\right\}>0 \\
\operatorname{Re} \frac{\beta|d| z+\frac{\Gamma(c)}{\Gamma(a)} \sum_{k=2}^{\infty} \frac{\Gamma(a+k)}{\Gamma(c+k) \Gamma(\lambda+k+1)}(k+\lambda-1+\beta|d|)[1+\alpha(k+\lambda-1)] a_{k+\lambda} z^{k+\lambda}}{z+\frac{\Gamma(c)}{\Gamma(a)} \sum_{k=2}^{\infty} \frac{\Gamma(a+k)}{\Gamma(c+k) \Gamma(\lambda+k+1)}[1+\alpha(k+\lambda-1)] a_{k+\lambda} z^{k+\lambda}}>0 .
\end{gathered}
$$

The inequality can be written in the following form considering $\operatorname{Re}\left(-e^{i \theta}\right) \geq-\left|e^{i \theta}\right|=-1$,

$$
\frac{\beta|d| r-\frac{\Gamma(c)}{\Gamma(a)} \sum_{k=2}^{\infty} \frac{\Gamma(a+k)}{\Gamma(c+k) \Gamma(\lambda+k+1)}(k+\lambda-1+\beta|d|)[1+\alpha(k+\lambda-1)] a_{k+\lambda} r^{k+\lambda}}{r-\frac{\Gamma(c)}{\Gamma(a)} \sum_{k=2}^{\infty} \frac{\Gamma(a+k)}{\Gamma(c+k) \Gamma(\lambda+k+1)}[1+\alpha(k+\lambda-1)] a_{k+\lambda} r^{k+\lambda}}>0 .
$$

Applying the mean value theorem for $r \rightarrow 1^{-}$, we find the inequality (6).

The proof of Theorem 1 is complete.

Corollary 1. Considering $f \in \mathcal{D} \oplus(d, \alpha, \beta, a, c, \lambda)$, the following inequalities can be written:

$$
a_{k+\lambda} \leq \frac{\beta|d| \Gamma(a) \Gamma(c+k) \Gamma(\lambda+k+1)}{\Gamma(c) \Gamma(a+k)(k+\lambda-1+\beta|d|)[1+\alpha(k+\lambda-1)]}, \quad k \geq 2 .
$$

\section{Distortion Results for Class $\mathcal{D} \oplus(d, \alpha, \beta, a, c, \lambda)$}

Theorem 2. The following inequalities are satisfied by any function $f \in \mathcal{D} \oplus(d, \alpha, \beta, a, c, \lambda)$ for $|z|=r<1$ :

$$
\begin{gathered}
r-\frac{c(c+1) \beta|d| \Gamma(\lambda+3)}{a(a+1)(\lambda+1+\beta|d|)[1+\alpha(\lambda+1)]} r^{2} \leq|f(z)| \\
\leq r+\frac{c(c+1) \beta|d| \Gamma(\lambda+3)}{a(a+1)(\lambda+1+\beta|d|)[1+\alpha(\lambda+1)]} r^{2} .
\end{gathered}
$$


The equality is true for

$$
f(z)=z+\frac{c(c+1) \beta|d| \Gamma(\lambda+3)}{a(a+1)(\lambda+1+\beta|d|)[1+\alpha(\lambda+1)]} z^{2} .
$$

Proof. Given that $f \in \mathcal{D} \oplus(d, \alpha, \beta, a, c, \lambda)$ from Equation (6) and since

$$
\frac{\Gamma(a+k)}{\Gamma(c+k) \Gamma(\lambda+k+1)}(k+\lambda-1+\beta|d|)[1+\alpha(k+\lambda-1)]
$$

is increasing and positive for $j \geq 2$, then we have

$$
\begin{gathered}
\frac{a(a+1)(\lambda+1+\beta|d|)[1+\alpha(\lambda+1)]}{c(c+1) \beta|d| \Gamma(\lambda+3)} \sum_{k=2}^{\infty} a_{k+\lambda} \leq \\
\sum_{k=2}^{\infty} \frac{\Gamma(a+k)}{\Gamma(c+k) \Gamma(\lambda+k+1)}(k+\lambda-1+\beta|d|)[1+\alpha(k+\lambda-1)] a_{k+\lambda} \leq \beta|d| \frac{\Gamma(a)}{\Gamma(c)},
\end{gathered}
$$

which is equivalent to,

$$
\sum_{k=2}^{\infty} a_{k+\lambda} \leq \frac{c(c+1) \beta|d| \Gamma(\lambda+3)}{a(a+1)(\lambda+1+\beta|d|)[1+\alpha(\lambda+1)]} .
$$

We obtain this using the properties of modulus function for

$$
\begin{gathered}
f(z)=z+\sum_{k=2}^{\infty} a_{k} z^{k} \\
r-r^{2} \sum_{k=2}^{\infty} a_{k} \leq r-\sum_{k=2}^{\infty} a_{k} r^{k} \leq|z|-\sum_{k=2}^{\infty} a_{k}|z|^{k} \leq|f(z)| \\
\leq|z|+\sum_{k=2}^{\infty} a_{k}|z|^{k} \leq r+\sum_{k=2}^{\infty} a_{k} r^{k} \leq r+r^{2} \sum_{k=2}^{\infty} a_{k}
\end{gathered}
$$

and by (7), we find

$$
\begin{gathered}
r-\frac{c(c+1) \beta|d| \Gamma(\lambda+3)}{a(a+1)(\lambda+1+\beta|d|)[1+\alpha(\lambda+1)]} r^{2} \leq f(z) \\
\quad \leq r+\frac{c(c+1) \beta|d| \Gamma(\lambda+3)}{a(a+1)(\lambda+1+\beta|d|)[1+\alpha(\lambda+1)]} r^{2} .
\end{gathered}
$$

The proof of Theorem 2 is complete.

Theorem 3. The following inequalities are satisfied by the derivative of any function $f \in \mathcal{D} \oplus$ $(d, \alpha, \beta, a, c, \lambda)$ for $|z|=r<1$ :

$$
\begin{gathered}
-\frac{2 c(c+1) \beta|d| \Gamma(\lambda+3)}{a(a+1)(\lambda+1+\beta|d|)[1+\alpha(\lambda+1)]} r \leq\left|f^{\prime}(z)\right| \\
\leq \frac{2 c(c+1) \beta|d| \Gamma(\lambda+3)}{a(a+1)(\lambda+1+\beta|d|)[1+\alpha(\lambda+1)]} r .
\end{gathered}
$$

For

$$
f(z)=z+\frac{c(c+1) \beta|d| \Gamma(\lambda+3)}{a(a+1)(\lambda+1+\beta|d|)[1+\alpha(\lambda+1)]} z^{2},
$$

we have the equality. 
Proof. The following result is obtained by applying the properties of the modulus function:

$$
\begin{gathered}
f^{\prime}(z)=1+\sum_{k=2}^{\infty} k a_{k} z^{k-1} \\
1-\sum_{k=2}^{\infty} k a_{k}|z| \leq 1-\sum_{k=2}^{\infty} k a_{k}|z|^{k-1} \leq\left|f^{\prime}(z)\right| \leq 1+\sum_{k=2}^{\infty} k a_{k}|z|^{k-1} \leq 1+\sum_{k=2}^{\infty} k a_{k}|z| .
\end{gathered}
$$

Now, using relation (7), we obtain:

$$
\begin{gathered}
1-\frac{2 c(c+1) \beta|d| \Gamma(\lambda+3)}{a(a+1)(\lambda+1+\beta|d|)[1+\alpha(\lambda+1)]} r \leq\left|f^{\prime}(z)\right| \\
\quad \leq 1+\frac{2 c(c+1) \beta|d| \Gamma(\lambda+3)}{a(a+1)(\lambda+1+\beta|d|)[1+\alpha(\lambda+1)]} r .
\end{gathered}
$$

Hence, the proof is complete.

\section{Properties of Partial Sums of Functions from Class $\mathcal{D} \oplus(d, \alpha, \beta, a, c, \lambda)$}

Theorem 4. Consider a function $h$,

$$
h(z)=\sum_{i=1}^{m} \mu_{i} f_{i}(z), \quad \mu_{i} \geq 0, \sum_{i=1}^{m} \mu_{i}=1,
$$

defined by using functions $f_{i}, i=1,2, \ldots, m$, where

$$
f_{i}(z)=z+\sum_{k=2}^{\infty} a_{k, i} z^{k}, \quad a_{k, i} \geq 0
$$

with $f_{i} \in \mathcal{D} \oplus(d, \alpha, \beta, a, c, \lambda), i=1,2, \ldots, m$. Then, such a function $h \in \mathcal{D} \oplus(d, \alpha, \beta, a, c, \lambda)$.

Proof. Function $h$ has the following form:

$$
h(z)=\sum_{i=1}^{m} \mu_{m} z+\sum_{i=1}^{m} \sum_{k=2}^{\infty} \mu_{i} a_{k, i} z^{k}=z+\sum_{k=2}^{\infty} \sum_{i=1}^{m} \mu_{i} a_{k, i} z^{k} .
$$

Applying Theorem 1 for the functions $f_{i}(z), i=1,2, \ldots, m$, from the class $\mathcal{D} \oplus(d, \alpha$, $\beta, a, c, \lambda)$, we obtain

$$
\sum_{k=2}^{\infty} \frac{\Gamma(a+k)}{\Gamma(c+k) \Gamma(\lambda+k+1)}(k+\lambda-1+\beta|d|)[1+\alpha(k+\lambda-1)] a_{k+\lambda, i} \leq \beta|d| \frac{\Gamma(a)}{\Gamma(c)} .
$$

Thus, it is sufficient to prove that:

$$
\begin{gathered}
\frac{\Gamma(c)}{\Gamma(a)} \sum_{k=2}^{\infty} \frac{\Gamma(a+k)}{\Gamma(c+k) \Gamma(\lambda+k+1)}(k+\lambda-1+\beta|d|)[1+\alpha(k+\lambda-1)]\left(\sum_{i=1}^{m} \mu_{i} a_{k+\lambda, i}\right)= \\
\frac{\Gamma(c)}{\Gamma(a)} \sum_{i=1}^{m} \mu_{i} \sum_{k=2}^{\infty} \frac{\Gamma(a+k)}{\Gamma(c+k) \Gamma(\lambda+k+1)}(k+\lambda-1+\beta|d|)[1+\alpha(k+\lambda-1)] a_{k+\lambda, i} \\
\leq \sum_{i=1}^{m} \mu_{i} \beta|d|=\beta|d|,
\end{gathered}
$$

which completes the proof. 
Corollary 2. A function $h$ defined as

$$
h(z)=(1-\xi) f_{1}(z)+\xi f_{2}(z), \quad 0 \leq \zeta \leq 1,
$$

with $f_{i} \in \mathcal{D} \oplus(d, \alpha, \beta, a, c, \lambda)$, and $i=1,2$, belongs to class $\mathcal{D} \oplus(d, \alpha, \beta, a, c, \lambda)$.

Theorem 5. Let

$$
f_{1}(z)=z
$$

and

$$
f_{k}(z)=z+\frac{\beta|d| \Gamma(a) \Gamma(c+k) \Gamma(\lambda+k+1)}{\Gamma(c) \Gamma(a+k)(k+\lambda-1+\beta|d|)[1+\alpha(k+\lambda-1)]} z^{k+\lambda}, \quad k \geq 2 .
$$

The function $f$ belongs to the class $\mathcal{D} \oplus(d, \alpha, \beta, a, c, \lambda)$ if and only if it can be written in the following form:

$$
f(z)=\mu_{1} f_{1}(z)+\sum_{k=2}^{\infty} \mu_{k} f_{k}(z)
$$

where $\mu_{1} \geq 0, \mu_{k} \geq 0, k \geq 2$ and $\mu_{1}+\sum_{k=2}^{\infty} \mu_{k}=1$.

Proof. Suppose that $f$ can be written as

$$
\begin{gathered}
f(z)=\mu_{1} f_{1}(z)+\sum_{k=2}^{\infty} \mu_{k} f_{k}(z), \\
=z+\sum_{k=2}^{\infty} \frac{\beta|d| \Gamma(a) \Gamma(c+k) \Gamma(\lambda+k+1)}{\Gamma(c) \Gamma(a+k)(k+\lambda-1+\beta|d|)[1+\alpha(k+\lambda-1)]} \mu_{k} z^{k+\lambda} .
\end{gathered}
$$

Thus,

$$
\begin{gathered}
\sum_{k=2}^{\infty} \frac{\Gamma(c) \Gamma(a+k)(k+\lambda-1+\beta|d|)[1+\alpha(k+\lambda-1)]}{\beta|d| \Gamma(a) \Gamma(c+k) \Gamma(\lambda+k+1)} . \\
\frac{\beta|d| \Gamma(a) \Gamma(c+k) \Gamma(\lambda+k+1)}{\Gamma(c) \Gamma(a+k)(k+\lambda-1+\beta|d|)[1+\alpha(k+\lambda-1)]} \mu_{k}=\sum_{k=2}^{\infty} \mu_{k}=1-\mu_{1} \leq 1 .
\end{gathered}
$$

Hence, $f(z) \in \mathcal{D} \oplus(d, \alpha, \beta, a, c, \lambda)$.

Conversely, suppose that $f \in \mathcal{D} \oplus(d, \alpha, \beta, a, c, \lambda)$.

Considering

$$
\mu_{k}=\frac{\Gamma(c) \Gamma(a+k)(k+\lambda-1+\beta|d|)[1+\alpha(k+\lambda-1)]}{\beta|d| \Gamma(a) \Gamma(c+k) \Gamma(\lambda+k+1)} a_{k},
$$

and

$$
\mu_{1}=1-\sum_{k=2}^{\infty} \mu_{k}
$$

we obtain that

$$
f(z)=\mu_{1} f_{1}(z)+\sum_{k=2}^{\infty} \mu_{k} f_{k}(z)
$$

which completes the proof.

Corollary 3. The extreme points of the class $\mathcal{D} \oplus(d, \alpha, \beta, a, c, \lambda)$ are the functions

$$
f_{1}(z)=z
$$

and

$$
f_{j}(z)=z+\frac{\beta|d| \Gamma(a) \Gamma(c+k) \Gamma(\lambda+k+1)}{\Gamma(c) \Gamma(a+k)(k+\lambda-1+\beta|d|)[1+\alpha(k+\lambda-1)]} z^{k}, \quad k \geq 2 .
$$




\section{Relations of Inclusion Involving Class $\mathcal{D} \oplus(d, \alpha, \beta, a, c, \lambda)$ and Neighborhoods}

We define the $\delta$ - neighborhood of a function $f(z) \in \mathcal{A}$ by

$$
N_{\delta}(f)=\left\{g \in \mathcal{A}: g(z)=z+\sum_{k=2}^{\infty} b_{k} z^{k} \text { and } \sum_{k=2}^{\infty} k\left|a_{k}-b_{k}\right| \leq \delta\right\} .
$$

A particular case when $e(z)=z$

$$
N_{\delta}(e)=\left\{g \in \mathcal{A}: g(z)=z+\sum_{k=2}^{\infty} b_{k} z^{k} \text { and } \sum_{k=2}^{\infty} k\left|b_{k}\right| \leq \delta\right\} .
$$

A function $f \in \mathcal{A}$ belongs to the class $\mathcal{D} \oplus^{\zeta}(d, \alpha, \beta, a, c, \lambda)$ if there exists a function $h \in \mathcal{D} \oplus(d, \alpha, \beta, a, c, \lambda)$ such that

$$
\left|\frac{f(z)}{h(z)}-1\right|<1-\zeta, \quad z \in U, 0 \leq \zeta<1 .
$$

Theorem 6. If

$$
\frac{\Gamma(a+k)}{\Gamma(c+k) \Gamma(\lambda+k+1)}[1+\alpha(\lambda+k-1)] \geq \frac{\Gamma(a+2)}{\Gamma(c+2) \Gamma(\lambda+3)}[1+\alpha(\lambda+1)], \quad k \geq 2
$$

and

$$
\delta=\frac{2 \beta|d| c(c+1) \Gamma(\lambda+3)}{a(a+1)(\lambda+1+\beta|d|)[1+\alpha(\lambda+1)]},
$$

then

$$
\mathcal{D} \oplus(d, \alpha, \beta, a, c, \lambda) \subset N_{\delta}(e) .
$$

Proof. Consider $f \in \mathcal{D} \oplus(d, \alpha, \beta, a, c, \lambda)$. Using relation (6) of Theorem 1 and the condition

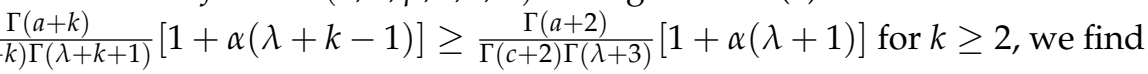

$$
\begin{gathered}
\frac{\Gamma(a+2)}{\Gamma(c+2) \Gamma(\lambda+3)}(\lambda+1+\beta|d|)[1+\alpha(\lambda+1)] \sum_{k=2}^{\infty} a_{k+\lambda} \leq \\
\sum_{k=2}^{\infty} \frac{\Gamma(a+k)}{\Gamma(c+k) \Gamma(\lambda+k+1)}(k+\lambda-1+\beta|d|)[1+\alpha(k+\lambda-1)] a_{k+\lambda} \leq \beta|d| \frac{\Gamma(a)}{\Gamma(c)},
\end{gathered}
$$

which implies

$$
\sum_{k=2}^{\infty} a_{k+\lambda} \leq \frac{\beta|d| c(c+1) \Gamma(\lambda+3)}{a(a+1)(\lambda+1+\beta|d|)[1+\alpha(\lambda+1)]} .
$$

Using relation (6) of Theorem 1 and relation (12), we obtain

$$
\begin{aligned}
& \frac{\beta|d| c(c+1) \Gamma(\lambda+3)}{a(a+1)(\lambda+1+\beta|d|)[1+\alpha(\lambda+1)]} \sum_{k=2}^{\infty} a_{k+\lambda} \leq \beta|d|, \\
& \frac{2 \beta|d| c(c+1) \Gamma(\lambda+3)}{a(a+1)(\lambda+1+\beta|d|)[1+\alpha(\lambda+1)]} \sum_{k=2}^{\infty} a_{k+\lambda} \leq 2 \beta|d| \\
& \sum_{k=2}^{\infty} k a_{k+\lambda} \leq \frac{2 \beta|d| c(c+1) \Gamma(\lambda+3)}{a(a+1)(\lambda+1+\beta|d|)[1+\alpha(\lambda+1)]}=\delta,
\end{aligned}
$$

and applying (9), we find $f \in N_{\delta}(e)$.

The proof of Theorem 6 is complete. 
Theorem 7. For $h \in \mathcal{D} \oplus(d, \alpha, \beta, a, c, \lambda)$ and

$$
\zeta=1-\frac{\delta}{2} \frac{a(a+1)(\lambda+1+\beta|d|)[1+\alpha(\lambda+1)]}{a(a+1)(\lambda+1+\beta|d|)[1+\alpha(\lambda+1)]-\beta|d| c(c+1) \Gamma(\lambda+3)}
$$

then

$$
N_{\delta}(h) \subset \mathcal{D} \oplus^{\zeta}(d, \alpha, \beta, a, c, \lambda)
$$

Proof. Assume that $f \in N_{\delta}(h)$, using (9), we find

$$
\sum_{k=2}^{\infty} k\left|a_{k}-b_{k}\right| \leq \delta
$$

which gives the coefficient inequality

$$
\sum_{k=2}^{\infty}\left|a_{k}-b_{k}\right| \leq \frac{\delta}{2}
$$

Applying relation (12) and considering that $h \in \mathcal{D} \oplus(d, \alpha, \beta, a, c, \lambda)$, we obtain

$$
\sum_{k=2}^{\infty} b_{k} \leq \frac{\beta|d| c(c+1) \Gamma(\lambda+3)}{a(a+1)(\lambda+1+\beta|d|)[1+\alpha(\lambda+1)]}
$$

Using (14) and (15), we find

$$
\begin{gathered}
\quad \frac{f(z)}{h(z)}-1 \mid \leq \frac{\sum_{k=2}^{\infty}\left|a_{k}-b_{k}\right|}{1-\sum_{k=2}^{\infty} b_{k}} \leq \frac{\delta}{2\left(1-\frac{\beta|d| c(c+1) \Gamma(\lambda+3)}{a(a+1)(\lambda+1+\beta|d|)[1+\alpha(\lambda+1)]}\right)} \\
\leq \frac{\delta}{2} \frac{a(a+1)(\lambda+1+\beta|d|)[1+\alpha(\lambda+1)]}{a(a+1)(\lambda+1+\beta|d|)[1+\alpha(\lambda+1)]-\beta|d| c(c+1) \Gamma(\lambda+3)}=1-\zeta .
\end{gathered}
$$

Using condition (11), we obtain that $f \in \mathcal{D} \oplus^{\zeta}(d, \alpha, \beta, a, c, \lambda)$, where $\zeta$ is given by (13).

\section{Starlikeness and Convexity Properties}

Theorem 8. Consider the function $f \in \mathcal{A}$ from the class $\mathcal{D} \oplus(d, \alpha, \beta, a, c, \lambda)$. The function $f$ is univalent starlike of order $\delta, 0 \leq \delta<1$, in $|z|<r_{1}$, where

$$
r_{1}=\inf _{k}\left\{\frac{(1-\delta) \Gamma(a+k) \Gamma(c)(k+\lambda-1+\beta|d|)[1+\alpha(k+\lambda-1)]}{\Gamma(a) \Gamma(c+k) \Gamma(\lambda+k+1) \beta|d|(2-\delta)}\right\}^{\frac{1}{k-1}} .
$$

When function $f$ has the form given by

$$
f_{k}(z)=z+\frac{\Gamma(a) \Gamma(c+k) \Gamma(\lambda+k+1) \beta|d|}{\Gamma(a+k) \Gamma(c)(k+\lambda-1+\beta|d|)[1+\alpha(k+\lambda-1)]} z^{k}, k \geq 2,
$$

and the result is sharp.

Proof. We show that

$$
\left|\frac{z f^{\prime}(z)}{f(z)}-1\right| \leq 1-\delta, \quad|z|<r_{1}
$$

Considering that

$$
\left|\frac{z f^{\prime}(z)}{f(z)}-1\right|=\left|\frac{\sum_{k=2}^{\infty}(k-1) a_{k} z^{k-1}}{1+\sum_{k=2}^{\infty} a_{k} z^{k-1}}\right| \leq \frac{\sum_{k=2}^{\infty}(k-1) a_{k}|z|^{k-1}}{1-\sum_{k=2}^{\infty} a_{k}|z|^{k-1}},
$$


to prove the theorem, we must show that

$$
\frac{\sum_{k=2}^{\infty}(k-1) a_{k}|z|^{k-1}}{1-\sum_{k=2}^{\infty} a_{k}|z|^{k-1}} \leq 1-\delta,
$$

which is equivalent to

$$
\sum_{k=2}^{\infty}(k-\delta) a_{k}|z|^{k-1} \leq 1-\delta,
$$

and applying Theorem 1, we have

$$
|z| \leq\left\{\frac{(1-\delta) \Gamma(a+k) \Gamma(c)(k+\lambda-1+\beta|d|)[1+\alpha(k+\lambda-1)]}{\Gamma(a) \Gamma(c+k) \Gamma(\lambda+k+1) \beta|d|(2-\delta)}\right\}^{\frac{1}{k-1}} .
$$

Hence, the proof is complete.

Theorem 9. Consider the function $f \in \mathcal{A}$ from the class $\mathcal{D} \oplus(d, \alpha, \beta, a, c, \lambda)$. The function $f$ is univalent convex of order $\delta, 0 \leq \delta \leq 1$, in $|z|<r_{2}$, where

$$
r_{2}=\inf _{k}\left\{\frac{(1-\delta) \Gamma(a+k) \Gamma(c)(k+\lambda-1+\beta|d|)[1+\alpha(k+\lambda-1)]}{2(k-\delta) \Gamma(a) \Gamma(c+k) \Gamma(\lambda+k+1) \beta|d|(2-\delta)}\right\}^{\frac{1}{k-1}} .
$$

When function $f$ has the form given by

$$
f_{k}(z)=z+\frac{\Gamma(a) \Gamma(c+k) \Gamma(\lambda+k+1) \beta|d|}{\Gamma(a+k) \Gamma(c)(k+\lambda-1+\beta|d|)[1+\alpha(k+\lambda-1)]} z^{k}, k \geq 2,
$$

and the result is sharp.

Proof. It is sufficient to prove that

$$
\left|\frac{z f^{\prime \prime}(z)}{\left.f^{\prime}(z)\right)}\right| \leq 1-\delta, \quad|z|<r_{2}
$$

Considering that

$$
\left|\frac{z f^{\prime \prime}(z)}{f^{\prime}(z)}\right|=\left|\frac{\sum_{k=2}^{\infty} k(k-1) a_{k} z^{k-1}}{1+\sum_{k=2}^{\infty} k a_{k} z^{k-1}}\right| \leq \frac{\sum_{k=2}^{\infty} k(k-1) a_{k}|z|^{k-1}}{1-\sum_{k=2}^{\infty} k a_{k}|z|^{k-1}},
$$

to prove the theorem, we must show that

$$
\begin{gathered}
\frac{\sum_{k=2}^{\infty} k(k-1) a_{k}|z|^{k-1}}{1-\sum_{k=2}^{\infty} k a_{k}|z|^{k-1}} \leq 1-\delta, \\
\sum_{k=2}^{\infty} k(k-\delta) a_{k}|z|^{k-1} \leq 1-\delta
\end{gathered}
$$

and applying Theorem 1, we find

$$
|z|^{k-1} \leq \frac{(1-\delta) \Gamma(a+k) \Gamma(c)(k+\lambda-1+\beta|d|)[1+\alpha(k+\lambda-1)]}{2(k-\delta) \Gamma(a) \Gamma(c+k) \Gamma(\lambda+k+1) \beta|d|},
$$

or

$$
|z| \leq\left\{\frac{(1-\delta) \Gamma(a+k) \Gamma(c)(k+\lambda-1+\beta|d|)[1+\alpha(k+\lambda-1)]}{2(k-\delta) \Gamma(a) \Gamma(c+k) \Gamma(\lambda+k+1) \beta|d|}\right\}^{\frac{1}{k-1}} .
$$

Hence, the proof is complete. 
Theorem 10. Consider the function $f \in \mathcal{A}$ from the class $\mathcal{D} \oplus(d, \alpha, \beta, a, c, \lambda)$. The function $f$ is univalent close-to-convex of order $\delta, 0 \leq \delta<1$, in $|z|<r_{3}$, where

$$
r_{3}=\inf _{k}\left\{\frac{(1-\delta) \Gamma(a+k) \Gamma(c)(k+\lambda-1+\beta|d|)[1+\alpha(k+\lambda-1)]}{k \Gamma(a) \Gamma(c+k) \Gamma(\lambda+k+1) \beta|d|}\right\}^{\frac{1}{k-1}} .
$$

The result is sharp for the function $f$ given by (16).

Proof. It is sufficient to prove that

$$
\left|f^{\prime}(z)-1\right| \leq 1-\delta, \quad|z|<r_{3}
$$

We obtain

$$
\left|f^{\prime}(z)-1\right|=\left|\sum_{k=2}^{\infty} k a_{k} z^{k-1}\right| \leq \sum_{k=2}^{\infty} k a_{k}|z|^{k-1},
$$

and thus $\left|f^{\prime}(z)-1\right| \leq 1-\delta$ if $\sum_{k=2}^{\infty} \frac{k a_{k}}{1-\delta}|z|^{k-1} \leq 1$. Using Theorem 1 , the inequality holds when

$$
|z|^{k-1} \leq \frac{(1-\delta) \Gamma(a+k) \Gamma(c)(k+\lambda-1+\beta|d|)[1+\alpha(k+\lambda-1)]}{k \Gamma(a) \Gamma(c+k) \Gamma(\lambda+k+1) \beta|d|}
$$

or

$$
|z| \leq\left\{\frac{(1-\delta) \Gamma(a+k) \Gamma(c)(k+\lambda-1+\beta|d|)[1+\alpha(k+\lambda-1)]}{k \Gamma(a) \Gamma(c+k) \Gamma(\lambda+k+1) \beta|d|}\right\}^{\frac{1}{k-1}} .
$$

Hence, the proof is complete.

\section{Conclusions}

A new operator $D_{z}^{-\lambda} \Phi(a, c)$ is introduced by convolution of the fractional integral of confluent hypergeometric function and an analytic function from class $\mathcal{A}$. The other original aspect of the paper is Definition 5, where a new class of analytic functions $\mathcal{D} \oplus(d, \alpha, \beta, a, c, \lambda)$ is defined using the operator $D_{z}^{-\lambda} \Phi(a, c)$. The next five sections of the paper contain the original results proved for the functions belonging to the newly defined class $\mathcal{D} \oplus(d, \alpha, \beta, a, c, \lambda)$. Necessary and sufficient conditions for class membership are presented, coefficient estimates are given for the functions in the class, and distortion theorems are proven for those functions. Closure theorems are proven regarding class $\mathcal{D} \oplus(d, \alpha, \beta, a, c, \lambda)$ and also that it is starlike, convex and close-to-convex for certain radii.

Further investigations are necessary for presenting examples of functions belonging to the new class introduced in this paper. As already explained in the Abstract, a new class of functions is introduced in this paper considering functions $f \in \mathcal{A}$. In further studies, functions $f \in \mathcal{A}_{n}, n>1$, might be investigated for defining new potentially interesting subclasses of analytic functions. As further directions of study on this class, subordination and superordination properties could be investigated involving functions from the class. The results could be adapted in view of quantum calculus aspects as seen in many recent papers, of which we only mention $[17,18]$.

The symmetry properties of the functions used in defining an equation or inequality could be studied to determine solutions with particular properties. Regarding the differential subordinations, some of which are inequalities, the study of special functions could provide interesting results given their symmetry properties. Studies on the symmetry properties of different types of functions associated with the concept of quantum computing could also be investigated in a future paper.

Author Contributions: Conceptualization, A.A.L. and G.I.O.; methodology, G.I.O.; software, A.A.L.; validation, A.A.L. and G.I.O.; formal analysis, A.A.L. and G.I.O.; investigation, A.A.L.; resources, G.I.O.; data curation, G.I.O.; writing—original draft preparation, A.A.L.; writing—review and editing, A.A.L. and G.I.O.; visualization, A.A.L.; supervision, G.I.O.; project administration, A.A.L.; funding acquisition, G.I.O. All authors have read and agreed to the published version of the manuscript. 
Funding: This research received no external funding.

Institutional Review Board Statement: Not applicable.

Informed Consent Statement: Not applicable.

Data Availability Statement: Not applicable.

Conflicts of Interest: The authors declare no conflicts of interest.

\section{References}

1. Alb Lupaş, A. Properties on a subclass of analytic functions defined by a fractional integral operator. J. Comput. Anal. Appl. 2019, $27,506-510$.

2. Alb Lupaş, A. Inequalities for Analytic Functions Defined by a Fractional Integral Operator. In Frontiers in Functional Equations and Analytic Inequalities; Anastassiou, G., Rassias, J., Eds.; Springer: Berlin/Heidelberg, Germany, 2020; pp. 731-745.

3. Owa, S. On the distortion theorems I. Kyungpook Math. J. 1978, 18, 53-59.

4. Owa, S.; Srivastava, H.M. Univalent and starlike generalized hypergeometric functions. Can. J. Math. 1987, $39,1057-1077$. [CrossRef]

5. Srivastava, H.M.; Bansal, M.; Harjule, P. A study of fractional integral operators involving a certain generalized multi-index Mittag-Leffler function. Math. Methods Appl. Sci. 2018, 41, 6108-6121. [CrossRef]

6. Cho, N.E.; Aouf, M.K.; Srivastava, R. The principle of differential subordination and its application to analytic and p-valent functions defined by a generalized fractional differintegral operator. Symmetry 2019, 11, 1083. [CrossRef]

7. Alb Lupaş, A.; Oros, G.I. Differential Subordination and Superordination Results Using Fractional Integral of Confluent Hypergeometric Function. Symmetry 2021, 13, 327. [CrossRef]

8. Ghanim, F.; Al-Shaqsi, K.; Darus, M.; Al-Janaby, H.F. Subordination Properties of Meromorphic Kummer Function Correlated with Hurwitz-Lerch Zeta-Function. Mathematics 2021, 9, 192. [CrossRef]

9. Ghanim, F.; Al-Janaby, H.F.; Bazighifan, O. Some New Extensions on Fractional Differential and Integral Properties for MittagLeffler Confluent Hypergeometric Function. Fractal Fract. 2021, 5, 143. [CrossRef]

10. Kanas, S.; Stankiewicz, J. Univalence of confluent hypergeometric function. Ann. Univ. Mariae Curie-Sklodowska 1998, 1, 51-56.

11. Oros, G.I. New Conditions for Univalence of Confluent Hypergeometric Function. Symmetry 2021, 13, 82. [CrossRef]

12. Miller, S.S.; Mocanu, P.T. Differential Subordinations. In Theory and Applications; Marcel Dekker Inc.: New York, NY, USA; Basel, Switzerland, 2000.

13. Juma, A.R.; Zirar H. Properties on a subclass of p-valent functions defined by new operator $V_{p}^{\lambda}$. Analele Univ. Oradea Fasc. Math 2014, 21, 73-82.

14. Yousef, A.T.; Salleh, Z.; Al-Hawary, T. On a class of p-valent functions involving generalized differential operator. Afr. Mat. 2021, 32, 275-287. [CrossRef]

15. Khan, B.; Srivastava, H.M.; Khan, N.; Darus, M.; Ahmad, Q.Z.; Tahir, M. Applications of Certain Conic Domains to a Subclass of q-Starlike Functions Associated with the Janowski Functions. Symmetry 2021, 13, 574. [CrossRef]

16. Al-Janaby, H.F.; Ghanim, F. A subclass of Noor-type harmonic p-valent functions based on hypergeometric functions. Kragujev. J. Math. 2021, 45, 499-519. [CrossRef]

17. Aouf, M.K.; Mostafa, A.O.; Elmorsy, R.E. Certain subclasses of analytic functions with varying arguments associated with q-difference operator. Afr. Mat. 2021, 32, 621-630. [CrossRef]

18. Srivastava, H.M.; Arif, M.; Raza, M. Convolution properties of meromorphically harmonic functions defined by a generalized convolution q-derivative operator. AIMS Math. 2021, 6, 5869-5885. [CrossRef] 